Article

\title{
Lower Triangular Factor-Based Fault Estimation and Fault-Tolerant Control for Descriptor Markovian Jump Systems with Multiple Faults
}

\author{
Xiaoming $\mathrm{Su}^{\dagger}$, Haoda Shi ${ }^{+}$(D) and Adiya Bao ${ }^{*}+$ (D) \\ School of Science, Shenyang University of Technology, Shenyang 110870, China; suxm@sut.edu.cn (X.S.); \\ shihaoda@smail.sut.edu.cn (H.S.) \\ * Correspondence: syeaady@outlook.com \\ † These authors contributed equally to this work.
}

check for updates

Citation: Su, X.; Shi, H.; Bao, A. Lower Triangular Factor-Based Fault Estimation and Fault-Tolerant Control for Descriptor Markovian Jump Systems with Multiple Faults. Symmetry 2022, 14, 382. https://doi.org/10.3390/ sym 14020382

Academic Editors: Jan Awrejcewicz, José A. Tenreiro Machado, José M. Vega, Hari Mohan Srivastava, Ying-Cheng Lai, Hamed Farokhi and Roman Starosta

Received: 7 December 2021

Accepted: 4 January 2022

Published: 15 February 2022

Publisher's Note: MDPI stays neutral with regard to jurisdictional claims in published maps and institutional affiliations.

Copyright: (c) 2022 by the authors. Licensee MDPI, Basel, Switzerland. This article is an open access article distributed under the terms and conditions of the Creative Commons Attribution (CC BY) license (https:// creativecommons.org/licenses/by/ $4.0 /)$.

\begin{abstract}
This paper considers the observer-based fault estimation and fault-tolerant control for descriptor Markovian jump systems (DMJSs). The goal of this paper is to estimate the actuator faults, sensor faults, and the state simultaneously, and then design a controller based on the estimation to stabilize the DMJS. Firstly, the state, actuator faults, and sensor faults are extended to new state variables to obtain an augmented system. Then, a lower triangular factor-based estimation observer (LTFEO) is proposed to estimate the state and multiple faults and to eliminate the influence of sensor faults. It is proved that the descriptor error system is derivative input-to-state stable (DISS) with respect to the derivative of the faults. Furthermore, based on the fault estimation, a fault-tolerant control scheme is proposed to guarantee the overall closed-loop system DISS. Finally, a numerical example is given to verify the effectiveness of the proposed estimation scheme and control strategy.
\end{abstract}

Keywords: descriptor markovian jump systems; fault estimation; lower triangular factor; faulttolerant control; derivative input-to-state stable

\section{Introduction}

Descriptor systems, also called generalized state-space systems, differential-algebraic systems, or singular systems, have been widely used due to their outstanding abilities in modeling practical engineering such as electrical circuits [1,2], biological systems [3], and mechanical systems $[4,5]$. Descriptor systems theory is an essential branch of modern control theory [1,6]. Meanwhile, Markovian jump systems (MJSs) are a special kind of multimodal stochastic hybrid system, and the modes can switch from one to another at different times [7,8]. Descriptor Markovian jump systems (DMJSs) can be modeled when descriptor systems experience sudden changes such as environmental mutation, component failures, and changes in subsystem interconnection. Accordingly, scholars have paid considerable attention to the analysis and study of the stability, robustness, and fault estimation on DMJSs. Numerous substantive results have been proposed in the past twenty years [9-13]. A super-twisting algorithm was developed to solve the integral sliding mode control problem for T-S fuzzy DMJSs in $[9,10]$, which investigated the issue of passivity analysis for the DMJSs using delayed partitioning. The robust stabilization problem of uncertain switching DMJSs was studied in [11,12], which considered the stochastic stabilization of a class of DMJSs with partly unknown transition rates. The robust $H_{\infty}$ control problem was investigated for DMJSs in [13], where the stochastic stability conditions of DMJSs were proposed.

In another area of research, the control systems are not completely reliable because faults may inevitably occur in normal system operations. It is usually caused by the aging of sensors and actuators, the wear and tear of internal components, and the abrupt changes in the working environment. These faults can dramatically affect the performance of 
the systems and even lead to significant accidents. Hence, it is of great significance to diagnose and estimate the faults from a stable system operation. Fault-tolerant control (FTC) is a control technique that can ensure the stable operation of the overall system in case of faults within the system $[14,15]$. Therefore, as a significant method of FTC, fault estimation has been widely studied $[16,17]$. There are plenty of methods and techniques that have been proposed for observer-based estimation, including the robust observer (RO) approach [18], adaptive observer $(\mathrm{AO})$ approach [19,20], and others [21-23]. In the existing fault estimation approaches, $\mathrm{AO}$ improves the estimation accuracy by considering adaptive law compared with the RO. In [19], the authors proposed an adaptive polytopic observer for linear parameter varying descriptor systems, where actuator faults were estimated by an adaptive algorithm. In [23], a more comprehensive form of estimation observer without sensor faults was proposed based on the lower triangular factor. However, faults on the measurement probably influence the closed-loop system performance, and thus it could not be neglected. As we all know, actuator faults and sensor faults often unavoidably appear in practical control systems simultaneously. The adverse effects caused by multiple faults need to be fully considered in the design process. Although the existing works have solved the issues of fault estimation and FTC, which focus on either actuator faults [15,23-27] or sensor faults [21,28]. So far, few related works have considered the case of the actuator faults and sensor faults appearing simultaneously. This case often occurs in practice, which is what motivated us to conduct this study.

In this paper, we study the fault estimation and FTC design problems for DMJS. Through appropriate design, the DMJS considered in this paper can achieve DISS property with respect to the derivative of the faults. Compared to existing literature, the main contributions of the paper are as follows:

(i) In contrast to the literature [15,21,23-28], in which either actuator faults or sensor faults were considered, this paper considers the case of the actuator faults, and sensor faults appear simultaneously. A lower triangular factor-based estimation observer (LTFEO) is designed to estimate the state, actuator faults, and sensor faults. This paper extends the LTFEO of non-singular fuzzy systems in [23] to DMJS. Based on the estimation, the influence of sensor faults is eliminated in the LTFEO such that the estimation of the state can be used in FTC design.

(ii) The designed LTFEO guarantees that the descriptor error system is derivative input-to-state stable (DISS) with respect to the derivative of the faults, which means the state of the descriptor error system asymptotically converges within the region defined by $|x| \leq \gamma\left(\|\dot{f}(t)\|_{[0, \infty)}\right)$, where $f(t)$ is the fault vector and $\gamma$ is the gain function. In addition, the state converges to zero when the derivative of the faults converges to zero. It is different from the existing results in [22,23]. The current literature [22,23] shows that the state of the error system is asymptotically stable only when the derivative of the faults is equal to zero.

The remainder of this paper is organized as follows: In Section 2, preliminaries and problem formulation are given. In Section 3, an LTFEO is proposed to simultaneously estimate the state, actuator faults, and sensor faults. Then, an observer-based FTC strategy is given. In Section 4, a numerical example is exhibited to verify the effectiveness of the proposed estimation method and control strategy. Section 5 summarizes the whole work of the paper.

Notations: $|\cdot|$ represents the Euclidean norm. $\mathcal{C}$ is the complex plane. $\|z\|_{\Delta}$ stands for the essential supremum of the truncation of $z$ on $\Delta$, that is $\|z\|_{\Delta}=\operatorname{ess} \sup \{|z(t)|: t \in \Delta\}$. $\lambda_{\max }(\cdot)$ and $\lambda_{\min }(\cdot)$ are the maximum and minimum eigenvalues of $(\cdot)$, respectively. diag (.) denotes the diagonal matrix. A function $\alpha: \mathbb{R}_{+} \rightarrow \mathbb{R}_{+}$is said to be a class $\mathcal{K}$ function if it is nondecreasing, continuous, and $\alpha(0)=0$; it is said to be a class $\mathcal{K}_{\infty}$ function if it is a class $\mathcal{K}$ function and satisfies $\alpha(s) \rightarrow \infty$ as $s \rightarrow \infty$. A function $\rho: \mathbb{R}_{+} \times \mathbb{R}_{+} \rightarrow \mathbb{R}_{+}$ is said to be a class $\mathcal{K} \mathcal{L}$ function if for each $t \in \mathbb{R}_{+}$, function $\rho(\cdot, t)$ is a class $\mathcal{K}$ function, and for each $s \in \mathbb{R}_{+}$, function $\rho(s, \cdot)$ is decreasing and satisfies $\lim _{t \rightarrow \infty} \rho(s, t)=0$. $\ell$ denotes the weak infinitesimal operator. $*$ denotes the symmetric parts of the symmetric matrices. $f^{k}\left(k \in \mathbb{N}_{+}\right)$denotes the $k$ th derivative of function $f$. 


\section{Problem Formulation and Preliminaries}

Consider the following DMJS with multiple faults:

$$
\left\{\begin{aligned}
E \dot{x}(t) & =A(r(t)) x(t)+B(r(t)) u(t)+D_{a}(r(t)) f_{a}(t), \\
y(t) & =C(r(t)) x(t)+D_{s}(r(t)) f_{s}(t) .
\end{aligned}\right.
$$

where $E \in \mathbb{R}^{n \times n}$ is a singular matrix with $\operatorname{rank}(E)=r<n, x(t) \in \mathbb{R}^{n}$ is the state, $u(t) \in \mathbb{R}^{d}$ is the control input, and $y(t) \in \mathbb{R}^{p}$ is the measurement output. $f_{a}(t) \in \mathbb{R}^{q}$ and $f_{s}(t) \in \mathbb{R}^{s}$ are actuator and sensor faults, respectively. $A(r(t)), B(r(t)), C(r(t)), D_{a}(r(t)), D_{s}(r(t))$ are real constant matrices with appropriate dimensions, $\{r(t), t \geq 0\}$ is a right-continuous Markov chain taking values from a finite set $S=\{1,2, \ldots, N\}$ with a generator $\Pi=\left(\pi_{i j}\right)(i, j \in S)$, referred to as the transition rate matrix:

$$
\operatorname{Pr}\{r(t+\Delta)=j \mid r(t)=i\}=\left\{\begin{array}{l}
\pi_{i j} \Delta+o(\Delta) \quad \text { if } i \neq j, \\
1+\pi_{i j} \Delta+o(\Delta) \text { if } i=j,
\end{array}\right.
$$

where $\Delta>0$ and $\lim _{\Delta \rightarrow 0} \frac{o(\Delta)}{\Delta}=0, \pi_{i j}$ is the switching rate from $i$ to $j$ and satisfies $\pi_{i j}>0$, with $i \neq j$ and $\pi_{i i}=-\sum_{j \neq i} \pi_{i j}<0$ for $\forall i, j \in S$.

For simplicity, the matrices $A(r(t)), B(r(t)), C(r(t)), D_{a}(r(t))$, and $D_{s}(r(t))$ are represented by $A_{i}, B_{i}, C_{i}, D_{a i}$, and $D_{s i}$, respectively. Now, System (1) can be rewritten as follows:

$$
\left\{\begin{aligned}
E \dot{x}(t) & =A_{i} x(t)+B_{i} u(t)+D_{a i} f_{a}(t), \\
y(t) & =C_{i} x(t)+D_{s i} f_{s}(t)
\end{aligned}\right.
$$

The following definitions, assumptions, and lemmas are given before presenting our main result:

Definition 1. In [1,6], the DMJS (10) is said to be

- regular-if there is a constant scalar s such that

$$
\operatorname{det}\left(s E-A_{i}\right) \neq 0, \forall i \in S
$$

or equivalently, the polynomial $\operatorname{det}\left(s E-A_{i}\right)$ is not identically zero for $\forall i \in S$.

- $\quad$ impulse free-if $\operatorname{deg}\left(\operatorname{det}\left(s E-A_{i}\right)\right)=\operatorname{rank}(E), \forall i \in S$.

Definition 2. Consider the following n-dimensional descriptor system:

$$
E \dot{x}=f(x, u)
$$

where $f(x, u): \mathbb{R}^{n} \times \mathbb{R}^{d} \rightarrow \mathbb{R}^{n}$ is the locally Lipschitz function. System (3) is said to be input-tostate stable (ISS) if for any initial state $x\left(t_{0}\right)=x_{0} \in \mathbb{R}^{n}$ and any measurable, locally essentially bounded input $u$, there exist $\beta \in \mathcal{K} \mathcal{L}$ and $\gamma \in \mathcal{K}$ such that the solution $x(t)$ satisfies the following equation:

$$
|x(t)| \leq \beta\left(\left|x_{0}\right|, t-t_{0}\right)+\gamma\left(\|u\|_{\left[t_{0}, t\right]}\right), \quad \forall t \geq t_{0}
$$

Definition 3. In [29], System (3) is said to be kth derivative input-to-state stable ( $D^{k} I S S$ ) if for any initial state $x\left(t_{0}\right)=x_{0} \in \mathbb{R}^{n}$ and any measurable, locally essentially bounded input $u$, there exist $\beta \in \mathcal{K} \mathcal{L}$ and some $\mathcal{K}$ functions $\gamma_{0}, \gamma_{1}, \ldots, \gamma_{k}$, such that the solution $x(t)$ satisfies the following equation:

$$
|x(t)| \leq \beta\left(\left|x_{0}\right|, t-t_{0}\right)+\gamma_{0}\left(\|u\|_{\left[t_{0}, t\right]}\right)+\gamma_{1}\left(\|\dot{u}\|_{\left[t_{0}, t\right]}\right)+\cdots+\gamma_{k}\left(\left\|u^{k}\right\|_{\left[t_{0}, t\right]}\right), \quad \forall t \geq t_{0}
$$

Remark 1. We say that System (3) is DISS when $k=1$. The kth derivative input-to-state stability reduces to input-to-state stability when $k=0$. Similar to the ISS property, the $k$ th derivative 
input-to-state stability becomes a globally asymptotic stability when $u$ is absent. The state vector $x$ converges to zero when $u^{i}(i=0,1, \ldots, k)$ converges to zero. The above properties correspond to a uniformly bounded-input bounded-state (UBIBS) property and a converging-input converging-state (CICS) property for nonlinear systems.

Assumption 1. System (2) is controllable, i.e., the following rank conditions hold:

$$
\begin{gathered}
\operatorname{rank}\left[s E-A_{i} \quad B_{i}\right]=n, \quad \forall s \in \mathcal{C}, \forall i=\{1,2, \ldots, N\} \\
\operatorname{rank}\left[E \quad B_{i}\right]=n
\end{gathered}
$$

Assumption 2. The derivative of actuator faults and sensor faults is locally essentially bounded.

Remark 2. The fault estimation problems are discussed in [22,23]. The authors assume that the derivative of the faults is bounded by a known constant. The error system is globally asymptotically stable only when the derivative of faults is identically equal to zero. Different from the existing literature, the designed LTFEO in this paper can estimate the unknown time-varying faults. The error system asymptotically converges to zero when the derivative of the faults converges to zero.

In the following, a lemma is given to present a sufficient condition of DISS.

Lemma 1. In [29], descriptor System (3) is considered. If there exist matrix $P$ such that $E^{T} P=$ $P^{T} E \geq 0$, and positive scalar $\kappa$ and $\gamma \in \mathcal{K}_{\infty}$ such that

$$
\begin{aligned}
V(x) & =x^{T} E^{T} P x \\
\ell V(x) f(x, u) & \leq-\kappa V(x)+\gamma(|\dot{u}|) .
\end{aligned}
$$

Then, System (3) is DISS, and the function $V$ is called a DISS Lyapunov function for System (3).

The following lemma is used in the proof of our main results.

Lemma 2. In [30], for any real matrices $M, N$, and $\Theta$ with an appropriate dimension, if $\Theta$ satisfies $\Theta^{T} \Theta \leq I$, then the following inequality holds:

$$
M \Theta N+(M \Theta N)^{T} \leq \sigma^{-1} M M^{T}+\sigma N^{T} N
$$

for any $\sigma>0$.

In what follows, an augmented system is constructed, whose state is composed of the original system state, actuator faults, and sensor faults.

First, we denote $f(t)=\left[\begin{array}{l}f_{a}(t) \\ f_{s}(t)\end{array}\right] \in \mathbb{R}^{m}$ with $m=q+s$, and correspondingly we have $\bar{D}_{a i}=\left[\begin{array}{ll}D_{a i} & 0\end{array}\right], \bar{D}_{s i}=\left[\begin{array}{ll}0 & D_{s i}\end{array}\right]$. Then, the original System (2) is equivalently transformed into the following system:

$$
\left\{\begin{aligned}
E \dot{x}(t) & =A_{i} x(t)+B_{i} u(t)+\bar{D}_{a i} f(t) \\
y(t) & =C_{i} x(t)+\bar{D}_{s i} f(t)
\end{aligned}\right.
$$

We make the following lower triangular factor-based linear transformation on system (10):

$$
\bar{x}(t)=F_{i}\left[\begin{array}{l}
x(t) \\
f(t)
\end{array}\right]
$$

where $F_{i}=\left[\begin{array}{cc}I_{n} & 0 \\ J & I_{m}\end{array}\right]$, and $J$ is an arbitrary constant matrix. 
Subsequently, an augmented system is described as follows:

$$
\left\{\begin{aligned}
\bar{E} \dot{\bar{x}}(t) & =F_{i} \bar{A}_{i} F_{i}^{-1} \bar{x}(t)+F_{i} \bar{B}_{i} u(t)+\bar{I}_{m} \dot{f}(t), \\
y(t) & =\bar{C}_{i} F_{i}^{-1} \bar{x}(t) .
\end{aligned}\right.
$$

where

$$
\bar{E}=\left[\begin{array}{cc}
E & 0 \\
J\left(E-I_{m}\right) & I_{m}
\end{array}\right], \bar{A}_{i}=\left[\begin{array}{cc}
A_{i} & \bar{D}_{a i} \\
0 & 0
\end{array}\right], \bar{B}_{i}=\left[\begin{array}{c}
B_{i} \\
0
\end{array}\right], \bar{C}_{i}=\left[\begin{array}{c}
C_{i} \\
\bar{D}_{s i}
\end{array}\right]^{T}, \bar{I}_{m}=\left[\begin{array}{c}
0 \\
I_{m}
\end{array}\right] .
$$

Assumption 3. System (12) is observable, i.e.,

$$
\operatorname{rank}\left[\begin{array}{c}
s \bar{E}-\tilde{A} \\
\tilde{C}
\end{array}\right]=n+m, \quad \forall s \in \mathcal{C}, \quad \forall i=\{1,2, \ldots, N\} .
$$

and

$$
\operatorname{rank}\left[\begin{array}{c}
\bar{E} \\
\tilde{C}
\end{array}\right]=n+m
$$

where

$$
\tilde{A}=F_{i} \bar{A}_{i} F_{i}^{-1}, \tilde{C}=\bar{C}_{i} F_{i}^{-1} .
$$

Remark 3. It should be noted that $F_{i}$ is a lower triangular matrix. Therefore, the observer with the factor is called the LTFEO. It is shown in [23] that LTFEO is more comprehensive, which can be reduced to the $R O$ in $[31,32]$ and estimate the faults quickly when $J=0$ and $\operatorname{rank}(E)=n$. In addition, the LTFEO can be reduced to the intermediate estimator in [22] when $J=-\omega \bar{D}_{a i}^{T}$ and the accuracy of fault estimation is improved, where $\omega$ is a positive scalar.

Remark 4. System (10) has been converted to the augmented DMJS (12), whose state consists of the original System (2) state, actuator faults, and sensor faults. Assumption 3 means that an observer can be designed for System (12) such that the state of the original System (2), actuator faults, and sensor faults can be estimated simultaneously.

\section{Main Results}

In this section, we propose a novel LTFEO to achieve a simultaneous estimation of $x(t), f_{s}(t)$, and $f_{a}(t)$ for System (2). Then, an effective FTC strategy is designed to stabilize the DMJS (2). The obtained overall closed-loop system is DISS with respect to the derivative of the faults, which means that the state flows in a large range if the derivative of the faults is large, and the state converges to zero if the derivative of the fault converges to zero.

\subsection{Lower Triangular Factor-Based Estimation Observer Design}

Inspired by Huang et al. [23], an improved observer for DMJS is proposed to estimate the state and faults. The observer is in the form of:

$$
\left\{\begin{aligned}
\bar{E} \dot{\hat{x}}(t) & =F_{i} \bar{A}_{i} F_{i}^{-1} \hat{x}(t)+F_{i} \bar{B}_{i} u(t)+L_{i}(y(t)-\hat{y}(t)), \\
\hat{y}(t) & =\bar{C}_{i} F_{i}^{-1} \hat{x}(t),
\end{aligned}\right.
$$

where $\hat{\bar{x}} \in \mathbb{R}^{n+m}$ is the estimation of the state of System (12), $\hat{y} \in \mathbb{R}^{p}$ is the observer output, and $L_{i}, i \in S=\{1,2, \ldots, N\}$ are the observer gain matrices to be designed.

Subsequently, the state estimation and fault estimation can also be derived as follows:

$$
\begin{gathered}
\hat{x}(t)=\bar{I}_{n} \hat{\bar{x}}(t) \\
\hat{f}_{a}(t)=\bar{I}_{q}\left[\bar{I}_{m}^{T} \hat{x}(t)-J \hat{x}(t)\right] \\
\hat{f}_{s}(t)=\bar{I}_{s}\left[\bar{I}_{m}^{T} \hat{x}(t)-J \hat{x}(t)\right]
\end{gathered}
$$


where

$$
\bar{I}_{n}=\left[\begin{array}{ll}
I_{n} & 0
\end{array}\right], \quad \bar{I}_{q}=\left[\begin{array}{ll}
I_{q} & 0
\end{array}\right], \quad \bar{I}_{s}=\left[\begin{array}{ll}
0 & I_{s}
\end{array}\right]
$$

Denote

$$
\begin{gathered}
e_{\bar{x}}(t)=\left[\begin{array}{lll}
e_{x}^{T}(t) & e_{f_{a}}^{T}(t) & e_{f_{s}}^{T}(t)
\end{array}\right]^{T}, \quad e_{x}(t)=x(t)-\hat{x}(t), \\
e_{f_{s}}(t)=f_{s}(t)-\hat{f}_{s}(t), \quad e_{f_{a}}(t)=f_{a}(t)-\hat{f}_{a}(t) .
\end{gathered}
$$

Then, the estimation error system can be obtained by using (12) and (13):

$$
\left\{\begin{aligned}
\bar{E} \dot{e}_{\bar{x}}(t) & =\left(F_{i} \bar{A}_{i} F_{i}^{-1}-L_{i} \bar{C}_{i} F_{i}^{-1}\right) e_{\bar{x}}(t)+\bar{I}_{m} \dot{f}(t) . \\
e_{y}(t) & =\bar{C}_{i} F_{i}^{-1} e_{\bar{x}}(t) .
\end{aligned}\right.
$$

Remark 5. The estimation of the state may not be accurate because of the appearance of the sensor faults $f_{s}(t)$. Thanks to $\hat{y}(t)=C_{i} \hat{x}(t)+D_{s i} \hat{f}_{s}(t)$, the influence of $f_{s}(t)$ can be eliminated in the designed LTFEO (13).

Remark 6. It is proved that the error system in $[22,33]$ is uniformly bounded. In this paper, we proved that the error System (17) is DISS with respect to the derivative of the faults, which means that the error System (17) preserves UBIBS and CICS properties with respect to the derivative of the faults.

In the following, we present the main result.

Theorem 1. The error System (17) is DISS if for each $i \in S$, given a positive scalar $\varepsilon_{1}$, there exists a set of nonsingular matrices $P_{i} \in \mathbb{R}^{n \times n}$ such that the following linear matrix inequalities hold:

$$
\begin{gathered}
\bar{E}^{T} P_{i}=P_{i}^{T} \bar{E} \geq 0, \\
\Pi_{1}=\left[\begin{array}{cc}
\Pi_{11} & P_{i} \bar{I}_{m} \\
* & -\varepsilon_{1} I
\end{array}\right]<0 .
\end{gathered}
$$

where

$$
\Pi_{11}=P_{i} F_{i} \bar{A}_{i} F_{i}^{-1}-\bar{L}_{i} \bar{C}_{i} F_{i}^{-1}+\left(F_{i} \bar{A}_{i} F_{i}^{-1}\right)^{T} P_{i}-\left(\bar{C}_{i} F_{i}^{-1}\right)^{T} \bar{L}_{i}^{T}+\sum_{j=1}^{N} \pi_{i j} \bar{E}^{T} P_{j} .
$$

Hence, by defining $\bar{L}_{i}=P_{i} L_{i}$, the observer gains $L_{i}$ can be solved.

Proof of Theorem 1. First of all, we prove that the system is regular and impulse-free for each $i \in S$. From (19), we know the following:

$$
P_{i} F_{i} \bar{A}_{i} F_{i}^{-1}-\bar{L}_{i} \bar{C}_{i} F_{i}^{-1}+\left(F_{i} \bar{A}_{i} F_{i}^{-1}\right)^{T} P_{i}-\left(\bar{C}_{i} F_{i}^{-1}\right)^{T} \bar{L}_{i}^{T}<-\sum_{j=1}^{N} \pi_{i j} \bar{E}^{T} P_{j}<0
$$

as $\operatorname{rank}(\bar{E})<n+m$, there exist non-singular matrices $\bar{U}$ and $\bar{V}$ such that $\bar{U} \bar{E} \bar{V}=\operatorname{diag}\left\{I_{n+m}, 0\right\}$. Moreover, we denote:

$$
\bar{U} \bar{A}_{i} \bar{V}=\left[\begin{array}{cc}
\bar{A}_{11 i} & \bar{A}_{12 i} \\
\bar{A}_{21 i} & \bar{A}_{22 i}
\end{array}\right], \bar{V}^{-T} P_{i} \bar{U}^{-1}=\left[\begin{array}{cc}
\bar{P}_{11 i} & \bar{P}_{12 i} \\
\bar{P}_{21 i} & \bar{P}_{22 i}
\end{array}\right] .
$$

Since $\bar{E}^{T} P_{i}=P_{i}^{T} \bar{E}$, it is not difficult to prove $\bar{P}_{11 i}^{T}=\bar{P}_{11 i}, \bar{P}_{21 i}=0$ and $\operatorname{det}\left(\bar{P}_{22 i}\right) \neq 0$. Then, pre- and post-multiplying (20) by $\bar{V}^{T}$ and $\bar{V}$, respectively, it follows that:

$$
\left[\begin{array}{lc}
\star & \star \\
\star & \bar{A}_{22 i}^{T} \bar{P}_{22 i}+\bar{P}_{22 i}^{T} \bar{A}_{22 i}
\end{array}\right]<0,
$$


where $\star$ are the matrix blocks we do not need to know. Then, we can easily obtain $\bar{A}_{22 i}^{T} \bar{P}_{22 i}+\bar{P}_{22 i}^{T} \bar{A}_{22 i}<0$ so that $\bar{A}_{22 i}$ is nonsingular, which implies that the system is regular and impulse-free.

Now, we are ready to prove that the system is DISS. Consider the following Lyapunov candidate functions:

$$
V_{i}\left(e_{\bar{x}}\right)=e_{\bar{x}}^{T}(t) \bar{E}^{T} P_{i} e_{\bar{x}}(t), \forall i \in S .
$$

Let $\ell$ be the weak infinitesimal operator [34] of $V_{i}\left(e_{\bar{x}}\right)$ along the trajectory of the system described in (12), then for each $i \in S$, it can be shown that:

$$
\begin{aligned}
\ell V_{i}\left(e_{\bar{x}}\right) & =\lim _{\Delta t \rightarrow 0^{+}} \frac{1}{\Delta t}\left\{E\left\{V\left(e_{\bar{x}}(t+\Delta t)\right), r(t+\Delta t) \mid e_{\bar{x}(t)}, r(t)=i\right\}-V\left(e_{\bar{x}(t)}, r(t)\right)\right\} \\
& =V_{t}\left(e_{\bar{x}(t)}, r(t)\right)+V_{e_{\bar{x}}}\left(e_{\bar{x}(t)}, r(t)\right)+\sum_{j=1}^{N} \pi_{i j} V\left(e_{\bar{x}(t)}, r(t)\right) \\
& =e_{\bar{x}}^{T}(t)\left[P_{i}\left(F_{i} \bar{A}_{i} F_{i}^{-1}-L_{i} \bar{C}_{i} F_{i}^{-1}\right)+\left(F_{i} \bar{A}_{i} F_{i}^{-1}-L_{i} \bar{C}_{i} F_{i}^{-1}\right)^{T} P_{i}\right] e_{\bar{x}}(t) \\
& +e_{\bar{x}}^{T}(t) \sum_{j=1}^{N} \pi_{i j} E^{T} P_{j} e_{\bar{x}}(t)+2 e_{\bar{x}}^{T}(t) P_{i} \bar{I}_{m} \dot{f}(t)
\end{aligned}
$$

Based on Lemma 2, the following inequalities always hold:

$$
2 e_{\bar{x}}^{T}(t) P_{i} \bar{I}_{m} \dot{f}(t) \leq \frac{1}{\epsilon_{1}} e_{\bar{x}}^{T}(t) P_{i} \bar{I}_{m} \bar{I}_{m}^{T} P_{i}^{T} e_{\bar{x}}(t)+\epsilon_{1}|\dot{f}(t)|^{2} .
$$

By substituting (24) in (23), it is obvious that:

$$
\begin{aligned}
\ell V_{i}\left(e_{\bar{x}}\right) \leq & e_{\bar{x}}^{T}(t)\left[P_{i}\left(F_{i} \bar{A}_{i} F_{i}^{-1}-L_{i} \bar{C}_{i} F_{i}^{-1}\right)+\left(F_{i} \bar{A}_{i} F_{i}^{-1}-L_{i} \bar{C}_{i} F_{i}^{-1}\right)^{T} P_{i}\right. \\
& \left.+\sum_{j=1}^{N} \pi_{i j} E^{T} P_{j}\right] e_{\bar{x}}(t)+\frac{1}{\epsilon_{1}} e_{\bar{x}}^{T}(t) P_{i} \bar{I}_{m} \bar{I}_{m}^{T} P_{i}^{T} e_{\bar{x}}(t)+\varepsilon_{1}|\dot{f}(t)|^{2} \\
& =e_{\bar{x}}^{T}(t) \Sigma_{1} e_{\bar{x}}(t)+\varepsilon_{1}|\dot{f}(t)|^{2},
\end{aligned}
$$

where

$$
\Sigma_{1}=P_{i}\left(F_{i} \bar{A}_{i} F_{i}^{-1}-L_{i} \bar{C}_{i} F_{i}^{-1}\right)+\left(F_{i} \bar{A}_{i} F_{i}^{-1}-L_{i} \bar{C}_{i} F_{i}^{-1}\right)^{T} P_{i}+\sum_{j=1}^{N} \pi_{i j} E^{T} P_{j}+\frac{1}{\epsilon_{1}} P_{i} \bar{I}_{m} \bar{I}_{m}^{T} P_{i}^{T}
$$

Furthermore, from (22), we have the following equation:

$$
V_{i}\left(e_{\bar{x}}\right) \leq \lambda_{\max }\left(\bar{E}^{T} P_{i}\right)\left|e_{\bar{x}}(t)\right|^{2}
$$

It is not hard to be found that if $\Sigma_{1}<0$, we have

$$
\begin{aligned}
\ell V_{i}\left(e_{\bar{x}}\right) & \leq \lambda_{\max }\left(\Sigma_{1}\right)\left|e_{\bar{x}}(t)\right|^{2}+\varepsilon_{1}|\dot{f}(t)|^{2} \\
& \leq-\kappa V_{i}\left(e_{\bar{x}}\right)+\varepsilon_{1}|\dot{f}(t)|^{2}
\end{aligned}
$$

where

$$
\kappa=\frac{\lambda_{\max }\left(\Sigma_{1}\right)}{\lambda_{\max }\left(\bar{E}^{T} P_{i}\right)} .
$$

Therefore, by Definition 2 and Lemma 1, it can be shown that the descriptor error System (17) is DISS with respect to the derivative of the faults. Moreover, by the Schur complement, we can find out that $\Sigma_{1}<0$ is equivalent to Condition (19) with $\bar{L}_{i}=P_{i} L_{i}$. The proof is completed. 


\subsection{Fault-Tolerant Control}

In this section, a novel FTC scheme is proposed to eliminate the influence of actuator faults, thereby stabilizing the closed-loop system.

Assumption 4. $\operatorname{rank}\left(B_{i}\right)=\operatorname{rank}\left(\left[\begin{array}{ll}B_{i} & D_{a i}\end{array}\right]\right), \forall i \in S$.

Lemma 3. In [35], for any Penrose-Moore inverse $B_{i}^{\dagger}$ of the matrix $B_{i}$, and any $D_{a i}$ that satisfies Assumption 4, we have $B_{i} B_{i}^{\dagger} D_{a i}=D_{a i}$.

An observer-based fault-tolerant controller is designed as follows:

$$
u(t)=K_{i} \hat{x}(t)-B_{i}^{\dagger} D_{a i} \hat{f}_{a}(t),
$$

where $K_{i}(i=1,2, \ldots, N)$ are the controller gains to be designed. $\hat{x}$ and $\hat{f}_{a}$ are defined in (14) and (15).

Substituting controller (29) into the system described in (2), we obtain the overall closed-loop system as:

$$
\begin{aligned}
E \dot{x}(t) & =A_{i} x(t)+B_{i} K_{i} \hat{x}(t)+D_{a i} f_{a}(t)-D_{a i} \hat{f}_{a}(t) \\
& =\left(A_{i}+B_{i} K_{i}\right) x(t)-B_{i} K_{i} e_{x}(t)+D_{a i} e_{f_{a}}(t) \\
& =\left(A_{i}+B_{i} K_{i}\right) x(t)+B_{i \mu} e(t),
\end{aligned}
$$

where

$$
B_{i \mu}=\left[\begin{array}{ll}
-B_{i} K_{i} & D_{a i}
\end{array}\right], \quad e(t)=\left[\begin{array}{ll}
e_{x}^{T}(t) & e_{f_{a}}^{T}(t)
\end{array}\right]^{T} .
$$

Next, we will propose an important theorem, which can guarantee that the overall closed-loop System (30) is DISS.

Theorem 2. The closed-loop System (30) is DISS if for each $i \in S$, given scalars $\varphi>0$ and $\psi>0$, there exist nonsingular and appropriately dimensioned matrices $T_{i}$, matrices $K_{i}$, such that the following matrix inequalities hold:

$$
\begin{gathered}
E^{T} T_{i}=T_{i}^{T} E \geq 0, \\
\Pi_{2}=\left[\begin{array}{cc}
T_{i} \Xi_{i}+\Xi_{i}^{T} T_{i}+\sum_{j=1}^{N} \pi_{i j} E^{T} T_{j}+\varphi E^{T} T_{i} & T_{i} B_{i \mu} \\
* & -\psi^{2} I
\end{array}\right]<0,
\end{gathered}
$$

where $\Xi_{i}=A_{i}+B_{i} K_{i}, B_{i \mu}$ is defined in (30).

Proof of Theorem 2. Choose the Lyapunov function as:

$$
V_{i}(x)=x^{T}(t) E^{T} T_{i} x(t) .
$$

The weak infinitesimal generator of $V_{i}(x)$ along the trajectory of System (30) is derived as follows:

$$
\begin{aligned}
\ell V_{i}(x) & =x^{T}(t)\left(T_{i} \Xi_{i}+\Xi^{T} T_{i}\right) x(t)+2 x^{T}(t) T_{i} B_{i \mu} e(t)+x^{T}(t) \sum_{j=1}^{N} \pi_{i j} E^{T} T_{j} x(t) \\
& \leq x^{T}(t)\left(T_{i} \Xi_{i}+\Xi_{i}^{T} T_{i}+\frac{1}{\psi^{2}} T_{i} B_{i \mu} B_{i \mu}^{T} T_{i}+\sum_{j=1}^{N} \pi_{i j} E^{T} T_{j}\right) x(t)+\psi^{2}|e(t)|^{2} .
\end{aligned}
$$


On the other hand, by the Schur complement, if (32) holds, it follows that:

$$
T_{i} \Xi_{i}+\Xi_{i}^{T} T_{i}+\sum_{j=1}^{N} \pi_{i j} E^{T} T_{j}+\frac{1}{\psi^{2}} T_{i} B_{i \mu} B_{i \mu}^{T} T_{i} \leq-\varphi E^{T} T_{i}
$$

It further follows that:

$$
\ell V_{i}(x) \leq-\varphi V_{3}(x)+\psi^{2}|e(t)|^{2} .
$$

Therefore, the closed-loop System (30) is ISS with respect to $e(t)$. Theorem 1 shows that the descriptor error System (17) is DISS with respect to the derivative of the faults. By the proof in [36], it can be easy to verify that a cascaded interconnected system composed of an ISS subsystem followed by a DISS subsystem is still DISS. Therefore, by (36) and (27), it means that the state of the overall closed-loop System (30) is also DISS with respect to the derivative of the faults. This completes the proof.

Since (32) is a nonlinear matrix inequality, it needs to be linearized by the following theorem.

Theorem 3. The state of the overall closed-loop System (30) is DISS if for each $i \in S$, given scalars $\varphi>0$ and $\psi>0$, there exist matrices $\bar{K}_{i}$, and $\Phi_{i}>0$, such that the following linear matrix inequalities hold:

$$
\begin{gathered}
E^{T} \Phi_{i}=\Phi_{i}^{T} E \geq 0, \\
\Pi_{3}=\left[\begin{array}{cc}
\bar{\Xi}_{i}+\bar{\Xi}_{i}^{T}+\sum_{j=1}^{N} \pi_{i j} E^{T} \Phi_{j}+\varphi E^{T} \Phi_{i} & B_{i \mu} \\
* & -\psi^{2} I
\end{array}\right]<0,
\end{gathered}
$$

with $\bar{\Xi}_{i}=A_{i} \Phi_{i}+B_{i} \bar{K}_{i}$.

Proof. Set $\Phi_{i}=T_{i}^{-1}$, and $\bar{K}_{i}=K_{i} \Phi_{i}$. If linear matrix inequalities (37) and (38) hold, we have the following equation:

$$
\Pi_{4}=\left[\begin{array}{cc}
\Xi_{i} \Phi_{i}+\Phi_{i} \Xi_{i}^{T}+\sum_{j=1}^{N} \pi_{i j} E^{T} \Phi_{j}+\varphi E^{T} \Phi_{i} & B_{i \mu} \\
* & -\psi^{2} I
\end{array}\right]<0,
$$

where $\Xi_{i}$ is defined in Theorem 2. Denote $\sigma=\operatorname{diag}\left\{T_{i}, I\right\}$, then, we can further deduce that $\Pi_{2}=\sigma \Pi_{4} \sigma<0$, that is, matrix inequality (32) holds. According to Theorem 2, the state of the overall closed-loop System (30) is DISS. This completes the proof.

\section{Simulation Examples}

In this section, a numerical example is given to verify the effectiveness of the proposed method. Consider a DMJS subject to actuator faults and sensor faults in the form of (1) with two modes. The parameters are as follows:

Mode 1:

$$
A_{1}=\left[\begin{array}{cc}
-0.34 & -1 \\
-0.2 & 4.87
\end{array}\right]
$$

Mode 2:

$$
A_{2}=\left[\begin{array}{cc}
-2 & 0.87 \\
1 & 1
\end{array}\right]
$$


The transition rate matrix is given as follows:

$$
\Pi=\left[\begin{array}{cc}
-0.9 & 0.9 \\
0.5 & -0.5
\end{array}\right]
$$

and other parameters are set as follows:

$$
\begin{gathered}
E=\left[\begin{array}{ll}
1 & 0 \\
0 & 0
\end{array}\right], J=\left[\begin{array}{cc}
1 & 1 \\
-0.1 & -0.1
\end{array}\right], B_{1}=B_{2}=D_{a 1}=D_{a 2}=\left[\begin{array}{c}
-1.43 \\
1
\end{array}\right], \\
C_{1}=C_{2}=\left[\begin{array}{cc}
0.8 & 1 \\
0 & 0.5
\end{array}\right], D_{s 1}=D_{s 2}=\left[\begin{array}{l}
0.5 \\
-1
\end{array}\right], \varepsilon_{1}=0.5, \psi=0.8565 .
\end{gathered}
$$

In this example, the actuator fault and sensor fault have the following forms:

$$
\begin{gathered}
f_{a}(t)=\left\{\begin{array}{lr}
\cos (5 t), & 0 \leq t<10 \\
e^{-0.5(t-10)} \cos (5 t), & 10 \leq t<50
\end{array}\right. \\
f_{s}(t)=\left\{\begin{array}{lr}
\sin (2 t), & 0 \leq t<4 \\
e^{-0.2(t-4)} \sin (5 t), & 4 \leq t<50
\end{array}\right.
\end{gathered}
$$

Next, the estimation observer and controller gains can be obtained by solving the linear matrix inequalities in Theorems 1 and 3 using the LMI toolbox in MATLAB. In addition, the differential equation is solved by Simulink module in MATLAB.

$$
\begin{gathered}
L_{1}=\left[\begin{array}{rr}
17.7593 & 10.2740 \\
-22.8709 & -13.0845 \\
-2.2039 & -1.6566 \\
-5.2407 & -1.1382
\end{array}\right], L_{2}=\left[\begin{array}{cr}
15.9582 & 5.5072 \\
-19.5472 & -6.9700 \\
-2.3499 & -1.3230 \\
-5.0178 & 0.0353
\end{array}\right], \\
K_{1}=\left[\begin{array}{ll}
2.7195 & -1.4435
\end{array}\right], K_{2}=\left[\begin{array}{ll}
2.3801 & -1.2598
\end{array}\right] .
\end{gathered}
$$

The initial value of the system is $x_{0}=\left[\begin{array}{ll}0.5 & 0.4\end{array}\right]^{T}$.

The actuator fault and sensor fault estimations using the proposed method are depicted in Figures 1 and 2, respectively. Figures 3 and 4 show the trajectories of the states and their estimation. One possible realization of the Markovian jumping mode is illustrated in Figure 5.

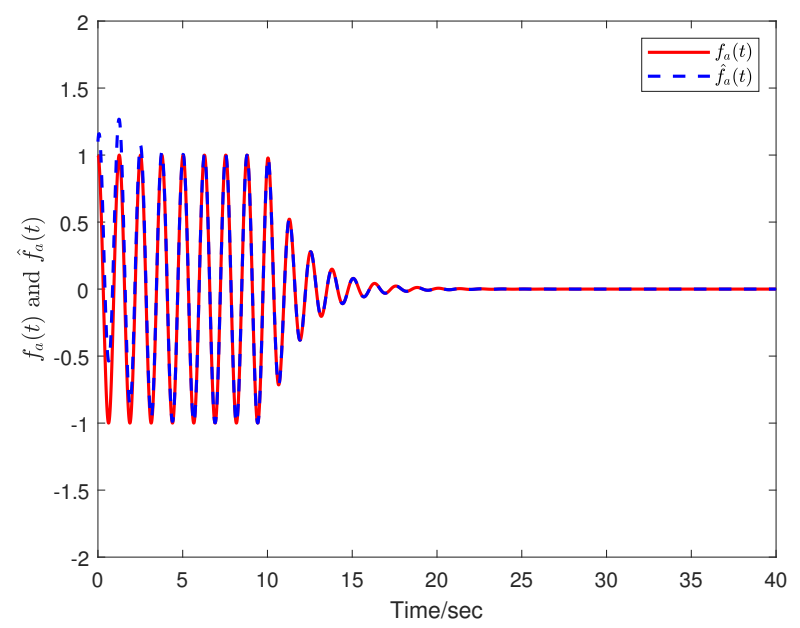

Figure 1. $f_{a}(t)$ and its estimation. 


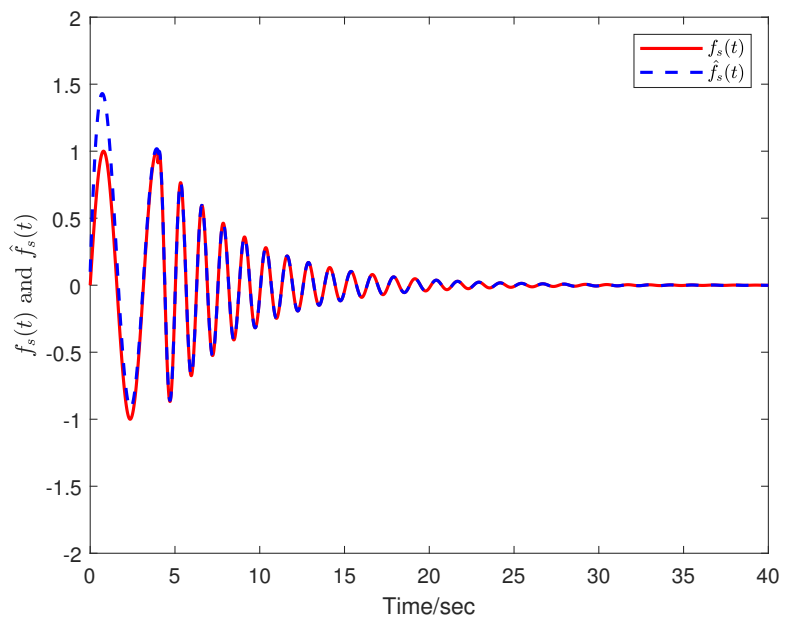

Figure 2. $f_{s}(t)$ and its estimation.

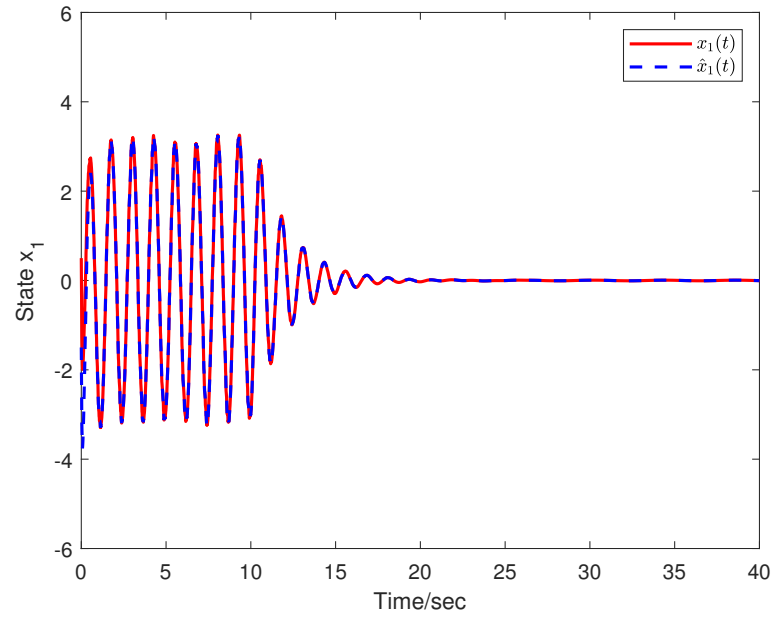

Figure 3. $x_{1}(t)$ and its estimation.

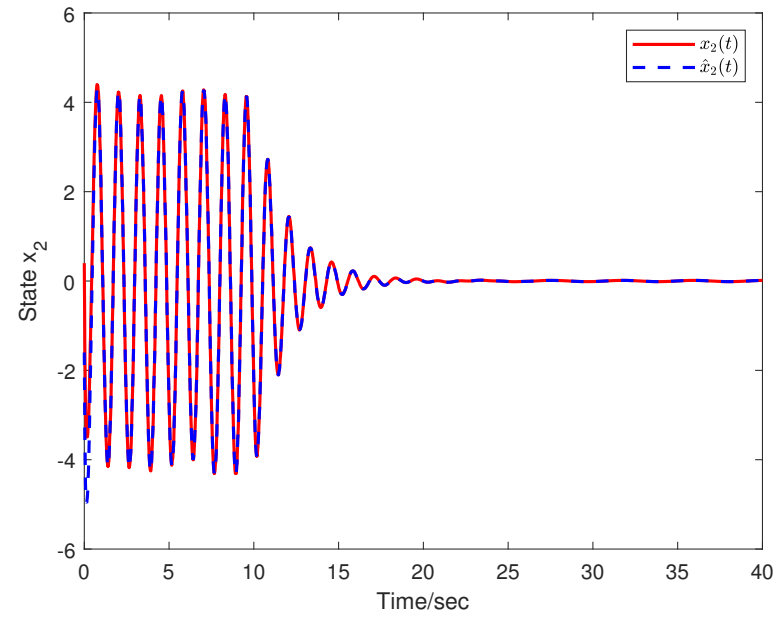

Figure 4. $x_{2}(t)$ and its estimation. 


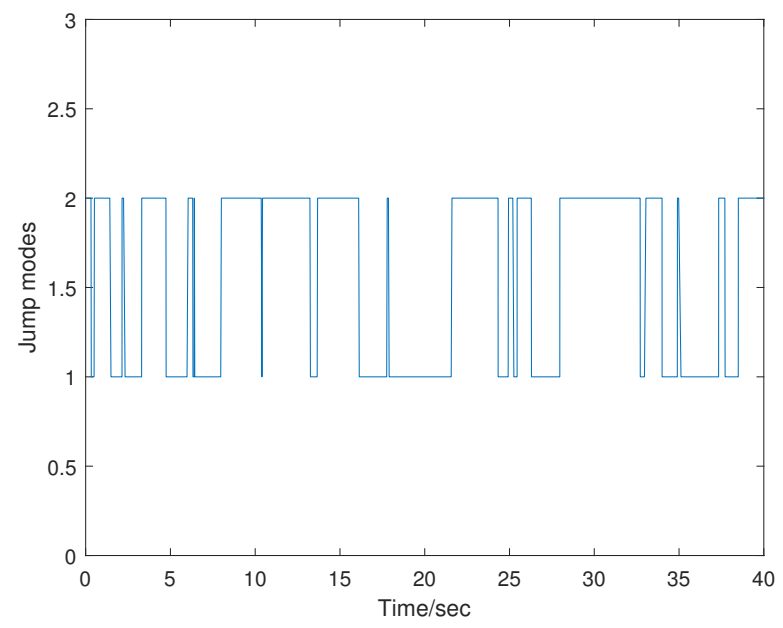

Figure 5. The jumping modes.

Figures 1-4 show that the state, actuator faults, and sensor faults can be estimated by the LTFEO (13). By defining the fault functions, it can easily be determined that their derivatives oscillate at first and then converge to zero. According to Figures 3 and 4, we can see that the state also oscillates at first and then converges to zero. It means that the proposed controller guarantees the DISS of the overall closed-loop system with respect to the derivative of the faults.

\section{Conclusions}

In this paper, the observer-based FTC problems for a class of descriptor Markovian jump systems with actuator faults and sensor faults are considered. An improved LTFEO is proposed to estimate the original system state, actuator faults, and sensor faults by constructing a new augmented system. The influence of the sensor faults is eliminated in the LTFEO such that the estimation of the original system state can be used for FTC design. It is proved that the error system is DISS with respect to the derivative of the faults. Compared with the FTC in the literature [23], an observer-based FTC strategy is proposed to guarantee that the state of the closed-loop system is ISS with respect to estimation error. It is shown that a cascaded, interconnected system composed of an ISS subsystem followed by a DISS subsystem is still DISS. Therefore, the state of the closed-loop system described in (30) is also DISS with respect to the derivative of the faults. Finally, a numerical example is given to verify the effectiveness of the proposed method. However, there are some limitations in this paper. For example, the controller designed in this paper cannot handle an asynchronous phenomenon of system mode and controller mode. Future work will have to focus on extending our designed methods to an asynchronous FTC for more complicated systems such as time-delay DMJSs with general uncertain transition rates.

Author Contributions: Writing—original draft, X.S. and H.S.; writing—review and editing, A.B. All authors have read and agreed to the published version of the manuscript.

Funding: This work was funded by the National Natural Science Foundation of China under Grant No. 61074005 and the Talent Project of the High Education of Liaoning Province (LR2012005).

Institutional Review Board Statement: Not applicable.

Informed Consent Statement: Not applicable.

Data Availability Statement: The data used to support the findings of this study were obtained directly from the simulation by the authors.

Acknowledgments: This research is supported by the National Natural Science Foundation of China under Grant No. 61074005 and the Talent Project of the High Education of Liaoning Province (LR2012005). 
Conflicts of Interest: The authors declare no conflict of interest.

\section{References}

1. Xu, S.; Lam, J. Robust Control and Filtering of Singular Systems; Springer: Berlin/Heidelberg, Germany, 2006.

2. Li, J.; Yang, G.H. Fuzzy descriptor sliding mode observer design: A canonical form-based method. IEEE Trans. Fuzzy Syst. 2019, 28, 2048-2062. [CrossRef]

3. Zhang, Q.; Liu, C.; Zhang, X. Complexity, Analysis and Control of Singular Biological Systems; Springer Science \& Business Media: Berlin/Heidelberg, Germany, 2012; Volume 421.

4. Duan, G.R. Analysis and Design of Descriptor Linear Systems; Springer Science \& Business Media: Berlin/Heidelberg, Germany, 2010; Volume 23.

5. Li, J.; Zhai, D. A descriptor regular form-based approach to observer-based integral sliding mode controller design. Int. J. Robust Nonlinear Control 2021, 31, 5134-5148. [CrossRef]

6. Dai, L. Singular Control Systems; Lecture Notes in Control and Information Sciences; Springer: Berlin, Germany, 1989 ; Volume 118.

7. Li, Y.; Yu, Z.; Liu, Y.; Ren, J. Stochastic Stabilization for Discrete-Time Markovian Jump Systems With Time-Varying Delay and Two Markov Chains Under Partly Known Transition Probabilities. IEEE Access 2021, 9, 26937-26947. [CrossRef]

8. Li, Y.; Sun, Q.; Ren, J.; Liu, Y. Sliding mode control of one-sided Lipschitz nonlinear Markovian jump systems with partially unknown transition rates. IEEE Access 2020, 8, 158306-158316. [CrossRef]

9. Li, J.; Zhang, Q.; Yan, X.G.; Spurgeon, S.K. Integral sliding mode control for Markovian jump T-S fuzzy descriptor systems based on the super-twisting algorithm. IET Control Theory Appl. 2017, 11, 1134-1143. [CrossRef]

10. Wu, Z.G.; Park, J.H.; Su, H.; Chu, J. Delay-dependent passivity for singular Markov jump systems with time-delays. Commun. Nonlinear Sci. Numer. Simul. 2013, 18, 669-681. [CrossRef]

11. Wang, G.L. Robust stabilization of singular Markovian jump systems with uncertain switching. Int. J. Control Autom. Syst. 2013, 11, 188-193. [CrossRef]

12. Li, L.; Zhang, Q. Finite-time $H_{\infty}$ control for singular Markovian jump systems with partly unknown transition rates. Appl. Math. Model. 2016, 40, 302-314. [CrossRef]

13. Lam, J.; Shu, Z.; Xu, S.; Boukas, E.K. Robust control of descriptor discrete-time Markovian jump systems. Int. J. Control 2007, 80, 374-385. [CrossRef]

14. Hamayun, M.T.; Edwards, C.; Alwi, H. Fault Tolerant Control Schemes Using Integral Sliding Modes; Springer: Berlin, Germany, 2016.

15. Chakravarty, A.; Mahanta, C. Actuator fault-tolerant control (FTC) design with post-fault transient improvement for application to aircraft control. Int. J. Robust Nonlinear Control 2016, 26, 2049-2074. [CrossRef]

16. Blanke, M.; Kinnaert, M.; Lunze, J.; Staroswiecki, M.; Schröder, J. Diagnosis and Fault-Tolerant Control; Springer: Berlin, Germany, 2006; Volume 2.

17. Ding, S.X. Model-Based Fault Diagnosis Techniques: Design Schemes, Algorithms, and Tools; Springer Science \& Business Media: Berlin, Germany, 2008.

18. Li, X.; Karimi, H.R.; Wang, Y.; Lu, D.; Guo, S. Robust fault estimation and fault-tolerant control for Markovian jump systems with general uncertain transition rates. J. Frankl. Inst. 2018, 355, 3508-3540. [CrossRef]

19. Rodrigues, M.; Hamdi, H.; Theilliol, D.; Mechmeche, C.; BenHadj Braiek, N. Actuator fault estimation based adaptive polytopic observer for a class of LPV descriptor systems. Int. J. Robust Nonlinear Control 2015, 25, 673-688. [CrossRef]

20. Huang, S.J.; Yang, G.H. Fault estimation for fuzzy delay systems: A minimum norm least squares solution approach. IEEE Trans. Cybern. 2016, 47, 2389-2399. [CrossRef]

21. Liu, M.; Shi, P.; Zhang, L.; Zhao, X. Fault-tolerant control for nonlinear Markovian jump systems via proportional and derivative sliding mode observer technique. IEEE Trans. Circuits Syst. Regul. Pap. 2011, 58, 2755-2764. [CrossRef]

22. Zhu, J.W.; Yang, G.H.; Wang, H.; Wang, F. Fault estimation for a class of nonlinear systems based on intermediate estimator. IEEE Trans. Autom. Control 2015, 61, 2518-2524. [CrossRef]

23. Huang, S.J.; Zhang, D.Q.; Guo, L.D.; Wu, L.B. Lower Triangle Factor-Based Fault Estimation and Fault Tolerant Control for Fuzzy Systems. IEEE Trans. Fuzzy Syst. 2019, 28, 2533-2542. [CrossRef]

24. Shi, P.; Liu, M.; Zhang, L. Fault-tolerant sliding-mode-observer synthesis of Markovian jump systems using quantized measurements. IEEE Trans. Ind. Electron. 2015, 62, 5910-5918. [CrossRef]

25. Jin, X. Adaptive fault-tolerant control for a class of output-constrained nonlinear systems. Int. J. Robust Nonlinear Control 2015, 25, 3732-3745. [CrossRef]

26. Yang, Y.; Yue, D. Distributed adaptive fault-tolerant control of pure-feedback nonlinear multi-agent systems with actuator failures. Neurocomputing 2017, 221, 72-84. [CrossRef]

27. Liu, M.; Ho, D.W.; Shi, P. Adaptive fault-tolerant compensation control for Markovian jump systems with mismatched external disturbance. Automatica 2015, 58, 5-14. [CrossRef]

28. Liu, M.; Cao, X.; Shi, P. Fault estimation and tolerant control for fuzzy stochastic systems. IEEE Trans. Fuzzy Syst. 2012, 21, 221-229. [CrossRef]

29. Angeli, D.; Sontag, E.D.; Wang, Y. Input-to-state stability with respect to inputs and their derivatives. Int. J. Robust Nonlinear Control.-Ifac-Affil. J. 2003, 13, 1035-1056. [CrossRef] 
30. Khargonekar, P.P.; Petersen, I.R.; Zhou, K. Robust stabilization of uncertain linear systems: Quadratic stabilizability and H/sup infinity/control theory. IEEE Trans. Autom. Control 1990, 35, 356-361. [CrossRef]

31. Zhang, K.; Jiang, B.; Staroswiecki, M. Dynamic output feedback-fault tolerant controller design for Takagi-Sugeno fuzzy systems with actuator faults. IEEE Trans. Fuzzy Syst. 2009, 18, 194-201. [CrossRef]

32. Huang, S.J.; Yang, G.H. Fault tolerant controller design for T-S fuzzy systems with time-varying delay and actuator faults: A K-step fault-estimation approach. IEEE Trans. Fuzzy Syst. 2014, 22, 1526-1540. [CrossRef]

33. Wang, G.; Yi, C. Fault estimation for nonlinear systems by an intermediate estimator with stochastic failure. Nonlinear Dyn. 2017, 89, 1195-1204. [CrossRef]

34. Dynkin, E.B. Functionals of Markov Processes: Markov Processes; Springer: Berlin/Heidelberg, Germany, 1965.

35. Li, X.; Zhu, F. Fault-tolerant control for Markovian jump systems with general uncertain transition rates against simultaneous actuator and sensor faults. Int. J. Robust Nonlinear Control 2017, 27, 4245-4274. [CrossRef]

36. Khalil, H.K. Nonlinear Systems, 3rd ed.; Prentice-Hall: Hoboken, NJ, USA, 2001. 